\title{
Children's rearfoot and midfoot motion while walking in school shoes
}

\author{
Caleb Wegener ${ }^{1 *}$, Richard Smith¹, Adrienne Hunt ${ }^{1}$, Benedicte Vanwanseele ${ }^{1}$, Andrew Greene ${ }^{1}$, Joshua Burns ${ }^{2}$ \\ From Australasian Podiatry Council Conference 2011 \\ Melbourne, Australia. 26-29 April 2011
}

\section{Background}

Parents, health professionals and shoe manufacturers expect that shoes will not impede a child's normal foot function or motor development. The aim of the current study was to determine the effect of school shoes on the rearfoot and midfoot motion of healthy children while walking.

\section{Methods}

Twelve children performed five walking trials at a self selected velocity while barefoot and wearing school shoes (Daytona, Clarks). A 10 camera 200Hz 3D motion analysis system (EVaRT5.0, MAC) was used to calculate marker trajectories. Force plate data were collected at $1000 \mathrm{~Hz}$ (Kistler ${ }^{\mathrm{TM}}$ ). Retro-reflective markers were attached to the right leg and to the foot/shoe at the navicular, $1^{\text {st }}$ and $5^{\text {th }}$ metatarsal heads and hallux. Rearfoot motion was measured with a wand marker cluster through a window in the shoe. A standing reference trial was used to embed segment axes and thence to calculate motion of the distal segment relative to the proximal segment. Data were normalised to the stance phase which was sub-divided from the anterior/posterior force data as: contact period (initial contact - maximum negative force); midstance (maximum negative force zero) and propulsion (positive force - toe-off).

\section{Results}

Five boys and seven girls participated in the study (mean age 9 years, range 5-13 years). During the contact period shoes decreased midfoot range of motion (ROM) in the frontal plane from $3.4^{\circ}$ to $1.7^{\circ}(\mathrm{p}=0.002)$ and in the transverse plane from $22.0^{\circ}$ to $11.6^{\circ}(\mathrm{p}<0.001)$. No significant difference in ROM occurred during midstance at either the rearfoot or midfoot. During propulsion shoes reduced rearfoot ROM in the frontal plane from $12.0^{\circ}$ to $9.6^{\circ}(\mathrm{p}=0.026)$ and midfoot $\mathrm{ROM}$ in the sagittal plan from $19.6^{\circ}$ to $10.8^{\circ}(\mathrm{p}<0.001)$ and in the transverse plan from $10.1^{\circ}$ to $4.3^{\circ}(\mathrm{p}<0.001)$.

\section{Conclusions}

Traditional school shoes restrict children's foot motion during walking particularly at the midfoot during the contact period and propulsion phases of gait. The medium and long-term impacts of these changes are the focus of further research. The impact of school shoes on foot motion should be considered when assessing the paediatric patient and evaluating the effect of shoe or in-shoe interventions.

\section{Author details}

${ }^{1}$ Discipline of Exercise and Sports Science, Faculty of Health Sciences, The University of Sydney, NSW, 1825, Australia. ${ }^{2}$ Faculty of Health Sciences, The University of Sydney/ Institute for Neuroscience and Muscle Research, The Children's Hospital at Westmead, Sydney, NSW, 2145, Australia.

Published: 20 May 2011

doi:10.1186/1757-1146-4-S1-O49

Cite this article as: Wegener et al:: Children's rearfoot and midfoot motion while walking in school shoes. Journal of Foot and Ankle Research 2011 4(Suppl 1):049.

\footnotetext{
* Correspondence: cweg6974@uni.sydney.edu.au

${ }^{1}$ Discipline of Exercise and Sports Science, Faculty of Health Sciences,

The University of Sydney, NSW, 1825, Australia

Full list of author information is available at the end of the article
} 